TRANSACTIONS OF THE

AMERICAN MATHEMATICAL SOCIETY

Volume 363, Number 2, February 2011, Pages 969-986

S 0002-9947(2010)05288-2

Article electronically published on September 21, 2010

\title{
A HOCHSCHILD COHOMOLOGY COMPARISON THEOREM FOR PRESTACKS
}

\author{
WENDY LOWEN AND MICHEL VAN DEN BERGH
}

\begin{abstract}
We generalize and clarify Gerstenhaber and Schack's "Special Cohomology Comparison Theorem". More specifically we obtain a fully faithful functor between the derived categories of bimodules over a prestack over a small category $\mathcal{U}$ and the derived category of bimodules over its corresponding fibered category. In contrast to Gerstenhaber and Schack we do not have to assume that $\mathcal{U}$ is a poset.
\end{abstract}

\section{INTRODUCTION}

Throughout $k$ is a commutative base ring. In 2, 3, 4, Gerstenhaber and Schack study deformation theory and Hochschild cohomology of presheaves of algebras. For a presheaf $\mathcal{A}$ of $k$-algebras on a small category $\mathcal{U}$, the corresponding Hochschild cohomology is defined a: 1

$$
\operatorname{HH}^{n}(\mathcal{A})=\operatorname{Ext}_{\operatorname{Bimod}(\mathcal{A})}^{n}(\mathcal{A}, \mathcal{A})
$$

To $\mathcal{A}$ Gerstenhaber and Schack associate a single algebra $\mathcal{A}$ !, and a functor

$$
(-) !: \operatorname{Bimod}(\mathcal{A}) \rightarrow \operatorname{Bimod}(\mathcal{A} !)
$$

which sends $\mathcal{A}$ to $\mathcal{A}$ ! and preserves Ext. It follows in particular that $\operatorname{HH}^{n}(\mathcal{A}) \cong$ $\mathrm{HH}^{n}(\mathcal{A} !)$. As (-)! does not preserve injectives nor projectives the fact that it preserves Ext is not at all a tautology.

In fact the construction of $\mathcal{A}$ ! and the proof of the preservation of Ext are rather difficult and proceed in several steps. The first step covers the case that $\mathcal{U}$ is a poset. In that case $\mathcal{A}$ ! is simply $\prod_{V \in \mathcal{U}} \bigoplus_{V \leq U} \mathcal{A}(V)$. This part of the construction is the so-called Special Cohomology Comparison Theorem (SCCT). It is stated and proved in [4].

To cover the general case Gerstenhaber and Schack doubly subdivide $\mathcal{U}$, which transforms it into a poset. The General Cohomology Comparison Theorem (GCCT) states that this subdivision preserves Ext. The statement of this theorem is given in [3], but the proof has not been published.

Received by the editors May 31, 2009.

2000 Mathematics Subject Classification. Primary 16E40, 18D30.

Key words and phrases. Hochschild cohomology, fibered categories, special cohomology comparison theorem.

The first author is a postdoctoral fellow with the Fund of Scientific Research Flanders (FWO).

The second author is a director of research at the FWO.

${ }^{1}$ For applications in deformation theory one would only use this definition if $\mathcal{A}$ is flat over $k$. The "correct" definition is obtained by replacing $\mathcal{A}$ first by a suitable $k$-flat resolution.

(C)2010 American Mathematical Society Reverts to public domain 28 years from publication 
In this paper we will be concerned with the SCCT. Our methods may also be used to give new insight on the GCCT, but this will be covered elsewhere.

We now discuss our main result (in a less general setting than in the body of the paper for the purposes of exposition). To $\mathcal{A}$ we may associate a " $\mathcal{U}$-graded category" (see [5] or \$2.2 below) which has an associated category of "bimodules" $\operatorname{Bimod}_{\mathcal{U}}(\mathfrak{a})$. Our generalization of the SCCT is the following.

Theorem 1.1 (A restricted version of Theorem 4.1). There is a natural functor

$$
\Pi^{*}: \operatorname{Bimod}(\mathcal{A}) \rightarrow \operatorname{Bimod}_{\mathcal{U}}(\mathfrak{a})
$$

which has the property $\Pi^{*}(\mathcal{A})=\mathfrak{a}$ and which induces a fully faithful functor between the corresponding derived categories.

In case $\mathcal{U}$ is a poset this theorem quickly yields the SCCT in the version of Gerstenhaber and Schack (see \$5). Despite the fact that our result is more general our proof seems more direct than the one by Gerstenhaber and Schack as we are able to leverage some basic properties of natural systems 1 .

Our main application of Theorem 1.1 is that combined with [5, Thm. 3.27] it implies that the Hochschild cohomology of $\mathcal{A}$ controls the deformation theory of $\mathcal{A}$ as a $k$-linear prestack 2 (but not as a presheaf of $k$-algebras!). In fact, from Theorem 4.1 below it follows that a similar result is true if $\mathcal{A}$ is itself a $k$-linear prestack.

In an appendix we describe the essential image of $\Pi^{*}$ as the objects inverting certain maps between projectives. Theorem 1.1 may be translated into saying that $\Pi^{*}$ is obtained from a certain stably flat universal localization 8 of linear categories. In particular the $K$-theoretic results of [7, 8, apply.

Finally we mention that Theorem 1.1 (or rather its generalization, Theorem 4.1) is a key ingredient in [6].

\section{PREstacks AND GRAded CATEgories}

In this section we quickly recall the relation between fibered categories and prestacks in the $k$-linear setting. For full details we refer to [5, 11].

2.1. The classical formalism. We first recall the classical theory when there is no additive structure. Let $\mathcal{U}, \mathfrak{a}, \phi$ be respectively a small category, an arbitrary category and a functor $\phi: \mathfrak{a} \rightarrow \mathcal{U}$. For $A, B \in \mathfrak{a}$ and for $f: \phi(A) \rightarrow \phi(B)$ in $\mathcal{U}$ put $\mathfrak{a}_{f}(A, B)=\phi^{-1}(f)$. For $U \in \mathrm{Ob}(\mathcal{U})$, we define a subcategory $\mathfrak{a}_{U}$ of $\mathfrak{a}$ as follows: $\operatorname{Ob}\left(\mathfrak{a}_{U}\right)=\phi^{-1}(U)$ and for $A, B \in \phi^{-1}(U)$ we put $\mathfrak{a}_{U}(A, B)=\mathfrak{a}_{1_{U}}(A, B)$.

Instead of specifying the functor $\phi$ we may just as well specify the (possibly big) sets $\mathrm{Ob}\left(\mathfrak{a}_{U}\right)$ and for $A \in \mathrm{Ob}\left(\mathfrak{a}_{U}\right), B \in \mathrm{Ob}\left(\mathfrak{a}_{V}\right)$ the decompositions

$$
\mathfrak{a}(A, B)=\coprod_{f: U \rightarrow V} \mathfrak{a}_{f}(A, B) .
$$

This is what we will do in the sequel.

If $f: U \rightarrow V$, then an arrow $\delta \in \mathfrak{a}_{f}(A, B), A \in \mathfrak{a}_{U}, B \in \mathfrak{a}_{V}$ is called cartesian if left composing with $\delta$ defines an isomorphism $\mathfrak{a}_{g}(C, A) \rightarrow \mathfrak{a}_{f g}(C, B)$ for all $W \in \mathcal{U}$, $C \in \mathfrak{a}_{w}, g: W \rightarrow U$. Given $f, B$, a cartesian arrow is necessarily unique up to unique isomorphism.

\footnotetext{
${ }^{2} k$-linear prestacks over $\mathcal{U}$ were called "pseudofunctors" in $[5]$.
} 
We say that $\mathfrak{a}$ is fibered if for any $f: U \rightarrow V$ in $\mathcal{U}$ and any $B \in \mathfrak{a}_{V}$ there exists a cartesian arrow $\delta_{f, B}: A \rightarrow B$.

Given a fibered $\mathcal{U}$-category, the choice of cartesian morphisms $\delta_{f, B} \in \mathfrak{a}_{f}(A, B)$ for every $f, B$ is called a cleavage. We will always choose a normalized cleavage, i.e. one in which $\delta_{1_{V}, B}=\operatorname{Id}_{B}$. If we have chosen a cleavage, then the domain of $\delta_{f, B}$ is denoted by $f^{*} B$.

In this way for every $B$ we obtain a functor $f^{*}: \mathfrak{a}_{V} \rightarrow \mathfrak{a}_{U}$. For compositions $W \stackrel{g}{\rightarrow} U \stackrel{f}{\rightarrow} V$ we obtain natural isomorphisms $(f g)^{*} \cong g^{*} f^{*}$ which satisfy the usual compatibility for triple compositions. In other words $U \mapsto \mathfrak{a}_{U}$ defines a pseudofunctor $\mathfrak{a}: \mathcal{U} \rightarrow$ Cat. This pseudo-functor satisfies $\mathfrak{a}\left(1_{V}\right)=\operatorname{Id}_{\mathfrak{a}_{V}}$. We will call such a pseudo-functor normalized.

We now have functors of 2-categories

\{fibered $\mathcal{U}$-categories $\} \leftarrow\{$ fibered $\mathcal{U}$-categories with a normalized cleavage $\}$

$$
\rightarrow \text { \{normalized pseudo-functors } \mathcal{U} \rightarrow \text { Cat }\},
$$

where the first one is the forgetful functor and the second one is the construction outlined in the above paragraphs. The above discussion shows that the first functor is an equivalence and one easily verifies that the second one is an isomorphism. The inverse functor associates to a normalized pseudo-functor $\mathcal{A}: \mathcal{U} \rightarrow$ Cat the fibered category a such that $\mathfrak{a}_{U}=\mathcal{A}(U)$ and

$$
\mathfrak{a}_{f}(A, B)=\mathcal{A}(U)\left(A, f^{*} B\right)
$$

for $f: U \rightarrow V, A \in \mathfrak{a}_{U}, B \in \mathfrak{a}_{V}$. A normalized cleavage is given by defining $\delta_{f, B}$ as the identity map in $\mathfrak{a}_{f}\left(f^{*} B, B\right)=\mathcal{A}(U)\left(f^{*} B, f^{*} B\right)$.

2.2. Additive structure. Let $\mathfrak{a} \rightarrow \mathcal{U}$ be as above. Following 5 we say that $\mathfrak{a}$ is ( $k$-linear) $\mathcal{U}$-graded if the sets $\mathfrak{a}_{f}(A, B)$ are equipped with the structure of a $k$ module such that the compositions $\mathfrak{a}_{g}(B, C) \times \mathfrak{a}_{f}(A, B) \rightarrow \mathfrak{a}_{g f}(A, C)$ are $k$-bilinear. If $\mathfrak{a}$ is fibered and we run this additional structure through (2.1) we find that $\mathfrak{a}$ now corresponds to a normalized pseudo-functor $\mathcal{U} \rightarrow \operatorname{Cat}(k)$ (where Cat $(k)$ stands for the 2-category of $k$-linear categories) and that this correspondence is reversible.

Below we will call a normalized pseudo-functor $\mathcal{U} \rightarrow \operatorname{Cat}(k)$ a $k$-linear prestack on $\mathcal{U}$.

Remark 2.1. If we equip $\mathcal{U}$ with the trivial Grothendieck topology, then this use of the word "prestack" is consistent with standard terminology as the usual gluing condition on maps is empty. Since the same is true for objects we might even have talked about stacks instead of prestacks.

\section{Module AND Bimodule CATEGories}

In this section we introduce a number of (bi)module categories and relate them. The only non-formal result is Lemma 3.13 .

3.1. Modules over a $k$-linear prestack. Recall that if $\mathfrak{l}$ is a $k$-linear category, then a (right) $\mathfrak{l}$-module is by definition a $k$-linear contravariant functor $\mathfrak{l} \rightarrow \operatorname{Mod}(k)$. This may be generalized to $k$-linear prestacks. To do this consider the constant presheaf $\underline{\operatorname{Mod}}(k)$ as a prestack on $\mathcal{U}$. 
Definition 3.1. Let $\mathcal{A}$ be a $k$-linear prestack on $\mathcal{U}$. An $\mathcal{A}$-module is a morphism of prestacks $M: \mathcal{A}^{\text {op }} \longrightarrow \underline{\operatorname{Mod}}(k)$. More concretely, an $\mathcal{A}$-module consists of the following data:

- for every $U \in \mathcal{U}$, an $\mathcal{A}(U)$-module $M^{U}$;

- for every $u: V \longrightarrow U$, a morphism of $\mathcal{A}(U)$-modules $\rho^{u}: M^{U} \longrightarrow M^{V} u^{*}$; such that the following additional compatibilities hold:

- for every $u: V \longrightarrow U, v: W \longrightarrow V, \rho^{u v}$ equals the canonical composition

$$
M^{U} \longrightarrow M^{V} u^{*} \longrightarrow M^{W} v^{*} u^{*} \longrightarrow M^{W}(u v)^{*}
$$

- for every $U \in \mathcal{U}, \rho^{U}: M^{U} \longrightarrow M^{U} 1^{*}=M^{U}$ is the identity.

A morphism of $\mathcal{A}$-modules $\varphi: M \longrightarrow N$ consists of morphisms

$$
\varphi^{U}: M^{U} \longrightarrow N^{U}
$$

commuting with the $\rho^{u}$.

Modules over a $k$-linear prestack $\mathcal{A}$ and their morphisms constitute an abelian category $\operatorname{Mod}(\mathcal{A})$.

3.2. Bimodules over $k$-linear prestacks. Let $\mathcal{A}, \mathcal{B}$ be two $k$-linear prestacks. By definition an $\mathcal{A}$ - $\mathcal{B}$-bimodule is a module over $\mathcal{A}^{\text {op }} \otimes \mathcal{B}$. More concretely an $\mathcal{A}$ - $\mathcal{B}$-bimodule $M$ is given by $k$-modules

$$
M^{U}(B, A)
$$

for $U \in \mathrm{Ob}(\mathcal{U}), A \in \mathrm{Ob}\left(\mathcal{A}_{U}\right), B \in \mathrm{Ob}\left(\mathcal{B}_{U}\right)$ which vary covariantly in $A$ and contravariantly in $B$ together with compatible restriction morphisms

$$
\rho^{u}(B, A): M^{U}(B, A) \rightarrow M^{V}\left(u^{*} B, u^{*} A\right)
$$

for $u: V \rightarrow U$ in $\mathcal{U}$.

The abelian category of $\mathcal{A}$ - $\mathcal{B}$-bimodules is denoted by $\operatorname{Bimod}(\mathcal{A}, \mathcal{B})$.

3.3. Bimodules over a graded category. $\mathcal{U}$-graded categories are mild generalizations of linear categories. In particular they admit a natural notion of a bimodule.

Definition 3.2 ([5, Definition 2.9]). Let $\mathfrak{a}$ and $\mathfrak{b}$ be $\mathcal{U}$-graded categories. An $\mathfrak{a}$-b-bimodule $M$ consists of $k$-modules

$$
M_{u}(B, A)
$$

for $u: V \longrightarrow U, B \in \mathrm{Ob}\left(\mathfrak{b}_{V}\right), A \in \mathrm{Ob}\left(\mathfrak{a}_{U}\right)$ and "multiplication" morphisms

$$
\rho: \mathfrak{a}_{w}\left(A, A^{\prime}\right) \otimes M_{u}(B, A) \otimes \mathfrak{b}_{v}\left(B^{\prime}, B\right) \longrightarrow M_{w u v}\left(B^{\prime}, A^{\prime}\right)
$$

satisfying the natural associativity and identity axioms.

The abelian category of $\mathfrak{a}$-b-bimodules is denoted by $\operatorname{Bimod}_{\mathcal{U}}(\mathfrak{a}, \mathfrak{b})$.

3.4. Functors between bimodule categories. Let $\mathcal{A}$ and $\mathcal{B}$ be $k$-linear prestacks on $\mathcal{U}$ with associated fibered graded categories $\mathfrak{a}$ and $\mathfrak{b}$ (see 2.2 ).

There is a natural functor

$$
\Pi^{*}: \operatorname{Bimod}(\mathcal{A}, \mathcal{B}) \longrightarrow \operatorname{Bimod}_{\mathcal{U}}(\mathfrak{a}, \mathfrak{b})
$$

defined by

$$
\left(\Pi^{*} M\right)_{u}(B, A)=M^{V}\left(B, u^{*} A\right)
$$

for $u: V \rightarrow U, A \in \mathrm{Ob}(\mathcal{A}(U))=\mathrm{Ob}\left(\mathfrak{a}_{U}\right), B \in \mathrm{Ob}(\mathcal{B}(V))=\mathrm{Ob}\left(\mathfrak{b}_{V}\right)$. 
3.5. Fibered bimodules. In this section we identify the essential image of $\Pi^{*}$.

Definition 3.3. Let $\mathfrak{a}$ and $\mathfrak{b}$ be fibered $\mathcal{U}$-graded categories. An $\mathfrak{a}$-b-bimodule $M$ is called fibered if for one (and hence for every) cartesian morphism $\delta \in \mathfrak{a}_{u}\left(A, A^{\prime}\right)$ with $u: V \rightarrow U, A \in \mathfrak{a}_{V}, A^{\prime} \in \mathfrak{a}_{U}$ we have for every $v: W \longrightarrow V, B \in \mathfrak{b}_{W}$ that the map

$$
M_{v}(B, A) \longrightarrow M_{u v}\left(B, A^{\prime}\right)
$$

given by left multiplication (cf. (3.1) ) with $\delta$ is an isomorphism.

Let

$$
\operatorname{Bimod}_{\mathcal{U}}^{\mathrm{fib}}(\mathfrak{a}, \mathfrak{b}) \subseteq \operatorname{Bimod}_{\mathcal{U}}(\mathfrak{a}, \mathfrak{b})
$$

denote the full subcategory of fibered bimodules.

Proposition 3.4. Assume that $\mathfrak{a}$ and $\mathfrak{b}$ are obtained from $k$-linear prestacks $\mathcal{A}$ and $\mathcal{B}$. Then the functor $\Pi^{*}$ induces an equivalence of categories

$$
\operatorname{Bimod}(\mathcal{A}, \mathcal{B}) \longrightarrow \operatorname{Bimod}_{\mathcal{U}}^{\mathrm{fib}}(\mathfrak{a}, \mathfrak{b}) .
$$

In particular $\Pi^{*}$ is fully faithful.

Proof. Let $N \in \operatorname{Bimod}(\mathcal{A}, \mathcal{B})$. We first show that $\Pi^{*} N$ is a fibered bimodule. With the notation of Definition 3.3 we have to show that the composition map

$$
\left(\Pi^{*} N\right)_{v}(B, A) \longrightarrow\left(\Pi^{*} N\right)_{u v}\left(B, A^{\prime}\right)
$$

is an isomorphism. We may choose $A=u^{*} A^{\prime}$ and $\delta=\delta_{u, A^{\prime}}$.

By (3.2) we have

$$
\begin{aligned}
\left(\Pi^{*} N\right)_{v}(B, A) & =N^{W}\left(B, v^{*} A\right)=N^{W}\left(B, v^{*} u^{*} A^{\prime}\right), \\
\left(\Pi^{*} N\right)_{u v}\left(B, A^{\prime}\right) & =N^{W}\left(B,(u v)^{*} A^{\prime}\right) .
\end{aligned}
$$

Making explicit the various definitions we see that the composition map is derived from the isomorphism $v^{*} u^{*}=(u v)^{*}$ and hence is itself an isomorphism. Thus $\Pi^{*} N$ is fibered.

Conversely assume that $M$ is a fibered $\mathfrak{a}-\mathfrak{b}$ bimodule. Then we may define an $\mathcal{A}-\mathcal{B}$ bimodule $\Pi_{*} M$ via

$$
\left(\Pi_{*} M\right)^{U}(B, A)=M_{1_{U}}(B, A)
$$

for $A \in \mathrm{Ob}(\mathcal{A}(U))=\mathrm{Ob}\left(\mathfrak{a}_{U}\right), B \in \mathrm{Ob}(\mathcal{B}(U))=\mathrm{Ob}\left(\mathfrak{b}_{U}\right)$, where the restriction maps

$$
\begin{aligned}
M_{1_{U}}(B, A) \stackrel{\text { def }}{=}\left(\Pi_{*} M\right)^{U}(B, A) \\
\quad \stackrel{\rho^{u}}{\longrightarrow}\left(\Pi_{*} M\right)^{V}\left(u^{*} B, u^{*} A\right) \stackrel{\text { def }}{=} M_{1_{V}}\left(u^{*} B, u^{*} A\right) \stackrel{\text { fib. }}{=} M_{u}\left(u^{*} B, A\right)
\end{aligned}
$$

for $u: V \rightarrow U$ are given by right multiplication with the cartesian arrow $\delta_{u, B} \in$ $\mathfrak{a}_{u}\left(u^{*} B, B\right)$.

It is easy to see that $\Pi^{*}$ and $\Pi_{*}$ define quasi-inverse functors between $\operatorname{Bimod}(\mathcal{A}, \mathcal{B})$ and $\operatorname{Bimod}_{\mathcal{U}}^{\text {fib }}(\mathfrak{a}, \mathfrak{b})$.

In the sequel we work with the category $\operatorname{Bimod}_{\mathcal{U}}^{\mathrm{fib}}(\mathfrak{a}, \mathfrak{b}) \operatorname{instead}$ of $\operatorname{Bimod}(\mathcal{A}, \mathcal{B})$. The previous proposition shows that this is equivalent. We will denote the inclusion $\operatorname{Bimod}_{\mathcal{U}}^{\text {fib }}(\mathfrak{a}, \mathfrak{b}) \hookrightarrow \operatorname{Bimod}_{\mathcal{U}}(\mathfrak{a}, \mathfrak{b})$ by $I$. 
3.6. Relation with presheaves and natural systems on $\mathcal{U}$. Let $\mathfrak{a}$ and $\mathfrak{b}$ be fibered $\mathcal{U}$-graded categories equipped with a normalized cleavage. Assume that $M$ is a fibered $\mathfrak{a}$-b-bimodule. Pick $W \in \mathcal{U}, A \in \mathfrak{a}_{W}, B \in \mathfrak{b}_{W}$. Then $M$ induces a presheaf $\Psi_{A, B}^{*}(M)$ of $k$-modules over $\mathcal{U} / W$ as follows: for $g: V \rightarrow W$ in $\mathcal{U} / W$ put

$$
\Psi_{A, B}^{*}(M)(g)=M_{1_{V}}\left(g^{*} B, g^{*} A\right) \text {. }
$$

The restriction morphisms are obtained from the fact that $M$ is obtained from a bimodule over the prestacks corresponding to $\mathfrak{a}, \mathfrak{b}$ (by Proposition 3.4).

Concretely for $v: V^{\prime} \rightarrow V$ the restriction map

$$
\begin{aligned}
M_{1_{V}}\left(g^{*} B, g^{*} A\right) & \stackrel{\text { def }}{=} \Psi_{A, B}^{*}(M)(g) \\
& \stackrel{\rho^{v}}{\longrightarrow} \Psi_{A, B}^{*}(M)(g v) \stackrel{\text { def }}{=} M_{1_{V^{\prime}}}\left((g v)^{*} B,(g v)^{*} A\right) \stackrel{\text { fib. }}{=} M_{v}\left(v^{*} g^{*} B, g^{*} A\right)
\end{aligned}
$$

is given by right multiplying with the cartesian arrow $\delta_{v, g^{*} B} \in \mathfrak{b}_{v}\left(v^{*} g^{*} B, g^{*} B\right)$.

We think of $\Psi_{A, B}^{*}(M)$ as a $(A, W, B)$-local version of $M$. For use below we present a generalization of this construction to the case that $M$ is not necessarily fibered.

A natural system of $k$-modules on $\mathcal{U}$ in the sense of [1] is by definition a functor

$$
\operatorname{Fact}(\mathcal{U}) \longrightarrow \operatorname{Mod}(k)
$$

where $\operatorname{Fact}(\mathcal{U})$ is the category with the morphisms $u: V \longrightarrow U$ of $\mathcal{U}$ as objects, and morphisms from $u$ to $u^{\prime}$ given by diagrams

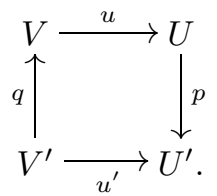

Natural systems on $\mathcal{U}$ constitute a category $\operatorname{Nat}(\mathcal{U})$.

Sending an arrow to its domain defines a functor

$$
\operatorname{Fact}(\mathcal{U}) \longrightarrow \mathcal{U}^{\text {op }}
$$

Hence from (3.4) we obtain a corresponding functor

$$
I: \operatorname{Pr}(\mathcal{U}) \longrightarrow \operatorname{Nat}(\mathcal{U})
$$

where $\operatorname{Pr}(\mathcal{U})$ is the category of $k$-linear presheaves on $\mathcal{U}$. Concretely for a presheaf $F$ and an arrow $u: V \rightarrow U$ we have

$$
(I F)(u)=F(V) .
$$

Clearly $I$ is fully faithful.

We define a natural system $\Phi_{A, B}^{*}(M)$ on $\mathcal{U} / W$ via

$$
\Phi_{A, B}^{*}(M)(u)=M_{u}\left((f u)^{*} B, f^{*} A\right),
$$

where $V \stackrel{u}{\rightarrow} U \stackrel{f}{\rightarrow} W$ represents an object in $\operatorname{Fact}(\mathcal{U} / W)$. 
Let us check that this has the right functoriality property. Consider the following morphism in $\operatorname{Fact}(\mathcal{U} / W)$ :

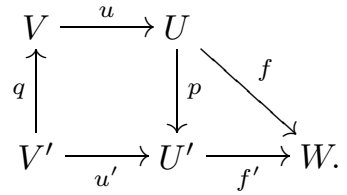

We have to produce a map

$$
M_{u}\left((f u)^{*} B, f^{*} A\right) \stackrel{\text { def }}{=} \Phi_{A, B}^{*}(M)(u) \longrightarrow \Phi_{A, B}^{*}(M)\left(u^{\prime}\right) \stackrel{\text { def }}{=} M_{u^{\prime}}\left(\left(f^{\prime} u^{\prime}\right)^{*} B, f^{*} A\right) .
$$

We have

$$
\begin{aligned}
M_{u}\left((f u)^{*} B, f^{*} A\right) & \cong M_{u}\left((f u)^{*} B, p^{*} f^{\prime *} A\right), \\
M_{u^{\prime}}\left(\left(f^{\prime} u^{\prime}\right)^{*} B, f^{\prime *} A\right) & \cong M_{p u q}\left(q^{*}(f u)^{*} B, f^{\prime *} A\right) .
\end{aligned}
$$

The required map is given by left multiplying with $\delta_{p, f^{\prime *} A} \in \mathfrak{a}_{p}\left(p^{*} f^{\prime *} A, f^{\prime *} A\right)$ and right multiplying with $\delta_{q,(u f)^{*} B} \in \mathfrak{b}_{v}\left(v^{*}(u f)^{*} B,(u f)^{*} B\right)$.

Proposition 3.5. Let $\mathfrak{a}, \mathfrak{b}$ be fibered $\mathcal{U}$-graded categories and let $W \in \mathcal{U}, A \in \mathfrak{a}_{W}$, $B \in \mathfrak{b}_{W}$. The following diagram is commutative:

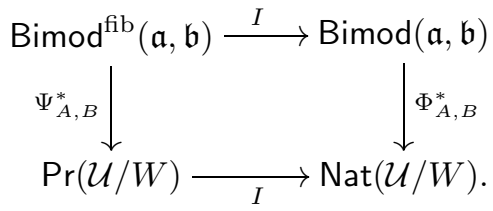

Proof. Easy.

Remark 3.6. Natural systems are a special case of bimodules over a fibered $\mathcal{U}$ graded category. More precisely, putting $\mathcal{A}=\mathcal{B}=\underline{k}$, the constant presheaf on $\mathcal{U}$, and $\mathfrak{k}$ the associated graded category, then natural systems on $\mathcal{U}$ are nothing but $\mathfrak{k}$-ḱ-bimodules.

3.7. Projective bimodules. Let $\mathfrak{a}, \mathfrak{b}$ be $\mathcal{U}$-graded categories. For $u: V \rightarrow U$, $B \in \mathfrak{b}_{V}, A \in \mathfrak{a}_{U}$ the exact functor

$$
\operatorname{Bimod}_{\mathcal{U}}(\mathfrak{a}, \mathfrak{b}) \longrightarrow \operatorname{Mod}(k): M \mapsto M_{u}(B, A)
$$

is representable by a projective object, which we denote by $P_{B, u, A}$. Concretely for $u^{\prime}: V^{\prime} \rightarrow U^{\prime}$ in $\mathcal{U}$ and $B \in \mathfrak{b}_{V^{\prime}}, A \in \mathfrak{a}_{U^{\prime}}$ we have

$$
\left(P_{B, u, A}\right)_{u^{\prime}}\left(B^{\prime}, A^{\prime}\right)=\bigoplus_{p u q=u^{\prime}} \mathfrak{b}_{q}\left(B^{\prime}, B\right) \otimes \mathfrak{a}_{p}\left(A, A^{\prime}\right) .
$$

An element

$$
b \otimes a \in \mathfrak{b}_{q}\left(B^{\prime}, B\right) \otimes \mathfrak{a}_{p}\left(A, A^{\prime}\right)
$$

may be interpreted in several equivalent ways.

- If $x \in M_{u}(B, A)$, then the corresponding map

$$
P_{B, u, A} \longrightarrow M
$$

sends $b \otimes a$ to bxa. 
- If we view $b \otimes a$ as a map

$$
P_{B^{\prime}, u^{\prime}, A^{\prime}} \longrightarrow P_{B, u, A}
$$

corresponding to $\operatorname{Id}_{B} \otimes \operatorname{Id}_{A} \in\left(P_{B, u, A}\right)_{u}(B, A)$, then the corresponding natural transformation from the functor

$$
M \mapsto M_{u}(B, A)
$$

to the functor

$$
M \mapsto M_{u^{\prime}}\left(B^{\prime}, A^{\prime}\right)
$$

is given by $m \mapsto a m b$.

From the second interpretation we obtain that if we have maps

$$
P_{B^{\prime \prime}, u^{\prime \prime}, A^{\prime \prime}} \stackrel{b^{\prime} \otimes a^{\prime}}{\longrightarrow} P_{B^{\prime}, u^{\prime}, A^{\prime}} \stackrel{b \otimes a}{\longrightarrow} P_{B, u^{\prime}, A},
$$

then the composition is given by $b b^{\prime} \otimes a^{\prime} a$.

For $u: V \rightarrow U, q: V^{\prime} \rightarrow V, p: U \rightarrow U^{\prime}, B \in \mathfrak{a}_{V}, A \in \mathfrak{a}_{U}, A^{\prime} \in \mathfrak{a}_{U^{\prime}}$ we have canonical maps

$$
\begin{aligned}
& \delta_{q}^{r}: P_{q^{*} B, u q, A} \longrightarrow P_{B, u, A}, \\
& \delta_{p}^{l}: P_{B, p u, A^{\prime}} \longrightarrow P_{B, u, p^{*} A^{\prime}},
\end{aligned}
$$

which are respectively associated to

$$
\begin{aligned}
& \delta_{q, B} \otimes \operatorname{Id}_{A} \in \mathfrak{b}_{q}\left(q^{*} B, B\right) \otimes \mathfrak{a}_{1_{U}}(A, A), \\
& \operatorname{Id}_{B} \otimes \delta_{p, A^{\prime}} \in \mathfrak{b}_{1_{V}}(B, B) \otimes \mathfrak{a}_{p}\left(p^{*} A^{\prime}, A^{\prime}\right) .
\end{aligned}
$$

These canonical maps will play an important role below.

Now let $W \in \mathcal{U}, A \in \mathfrak{a}_{W}, B \in \mathfrak{b}_{W}$. The functors $\Phi_{A, B}^{*}, \Psi_{A, B}^{*}$ introduced in $\$ 3.6$ are functors between Grothendieck categories commuting with products. Hence they have left adjoints which we denote respectively by $\Phi_{A, B, !}$ and $\Psi_{A, B, !}$. Since these functors have exact right adjoints they preserve projectives.

Lemma 3.7. For $V \stackrel{u}{\rightarrow} U \stackrel{f}{\rightarrow} W$ in $\mathcal{U} / W$, let $P_{u}$ be the projective natural system on $\mathcal{U} / W$ given by

(1) We have

$$
P_{u}=k \operatorname{Fact}(\mathcal{U} / W)(u,-) .
$$

$\Phi_{A, B, !}\left(P_{u}\right)=P_{(f u)^{*} B, u, f^{*} A}$.

(2) The morphism $P_{u^{\prime}} \rightarrow P_{u}$ corresponding to the morphism $u \rightarrow u^{\prime}$ in $\operatorname{Fact}(\mathcal{U} / W)$ given by

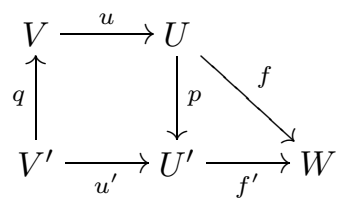

is sent under $\Phi_{A, B, !}$ to $\delta_{q}^{r} \delta_{p}^{l}=\delta_{p}^{l} \delta_{q}^{r}$.

Proof. To verify the first claim we compute for $M \in \operatorname{Bimod}_{\mathcal{U}}(\mathfrak{a}, \mathfrak{b})$,

$$
\begin{aligned}
\operatorname{Hom}\left(\Phi_{A, B, !}\left(P_{u}\right), M\right) & =\operatorname{Hom}\left(P_{u}, \Phi_{A, B}^{*} M\right) \\
& =\left(\Phi_{A, B}^{*} M\right)(u) \\
& =M_{u}\left((f u)^{*} B, f^{*} A\right) .
\end{aligned}
$$

The second claim is verified in a similar way. 
Remark 3.8. There is an alternative way to think about the projective objects $P_{B, u, A}$. Introduce the $k$-linear category $\mathfrak{t}=\mathfrak{a}^{\circ} \otimes \mathfrak{U} \mathfrak{b}$ (see [5], Definition 2.10) as follows:

$$
\mathrm{Ob}(\mathfrak{t})=\left\{(B, u, A) \mid u: V \rightarrow U \in \mathcal{U}, B \in \mathrm{Ob}\left(\mathfrak{b}_{V}\right), A \in \mathrm{Ob}\left(\mathfrak{a}_{U}\right)\right\}
$$

with

$$
\mathfrak{t}\left(\left(B^{\prime}, u^{\prime}, A^{\prime}\right),(B, u, A)\right)=\bigoplus_{u^{\prime}=p u q} \mathfrak{b}_{q}\left(B^{\prime}, B\right) \otimes \mathfrak{a}_{p}\left(A, A^{\prime}\right)
$$

with the obvious composition.

Then there is an isomorphism of categories

$$
\operatorname{Bimod}_{\mathcal{U}}(\mathfrak{a}, \mathfrak{b}) \longrightarrow \operatorname{Mod}(\mathfrak{t}): M \mapsto\left((B, u, A) \mapsto M_{u}(B, A)\right) .
$$

Under this isomorphism we have

$$
P_{B, u, A}=\mathfrak{t}(-,(B, u, A)) .
$$

If $W \in \mathcal{U}, A, B \in \mathfrak{b}_{W}$, then we have an associated functor

$$
\operatorname{Fact}(\mathcal{U} / W) \longrightarrow \mathfrak{t}^{\circ}:(V \stackrel{u}{\rightarrow} U \stackrel{f}{\rightarrow} W) \mapsto\left((f u)^{*} B, u, f^{*} A\right) .
$$

Hence we get a corresponding dual functor

$$
\operatorname{Mod}(\mathfrak{t}) \longrightarrow \operatorname{Nat}(\mathcal{U} / W) \text {. }
$$

Composing this with (3.7) we obtain a functor

$$
\operatorname{Bimod}_{\mathcal{U}}(\mathfrak{a}, \mathfrak{b}) \longrightarrow \operatorname{Nat}(\mathcal{U} / W)
$$

which turns out to be precisely $\Phi_{A, B}^{*}$. From this one easily obtains Lemma 3.7

3.8. Projective fibered bimodules. Let $\mathcal{A}, \mathcal{B}$ be $k$-linear prestacks on $\mathcal{U}$ with associated fibered categories $\mathfrak{a}, \mathfrak{b}$. If $W \in \mathcal{U}$ and $A \in \mathfrak{a}_{W}, B \in \mathfrak{b}_{W}$ the functor

$$
\operatorname{Bimod}_{\mathcal{U}}^{\mathrm{fib}}(\mathfrak{a}, \mathfrak{b}) \longrightarrow \operatorname{Mod}(k): M \mapsto M_{1_{W}}(B, A)
$$

is representable by a projective object $P_{B, W, A}^{\mathrm{fib}}$. Again concretely for $u^{\prime}: V \longrightarrow U$, $B^{\prime} \in \mathfrak{b}_{V}, A^{\prime} \in \mathfrak{a}_{U}$ we have

$$
\left(P_{B, W, A}^{\mathrm{fib}}\right)_{u^{\prime}}\left(B^{\prime}, A^{\prime}\right)=\bigoplus_{u: V \rightarrow W} \mathfrak{b}_{1_{V}}\left(B^{\prime}, u^{*} B\right) \otimes \mathfrak{a}_{u^{\prime}}\left(u^{*} A, A^{\prime}\right) .
$$

Remark 3.9. Again, there is an alternative way to think about the projective objects $P_{B, W, A}^{\mathrm{fib}}$. Let $\mathfrak{r}$ be the underlying linear category of the fibered graded category associated to $\mathcal{A} \otimes \mathcal{B}$. Concretely,

$$
\mathrm{Ob}(\mathfrak{r})=\{(B, W, A) \mid W \in \mathrm{Ob}(\mathcal{U}), B \in \mathrm{Ob}(\mathcal{B}(W)), A \in \mathrm{Ob}(\mathcal{A}(W))\}
$$

and

$$
\mathfrak{r}\left(\left(B^{\prime}, W^{\prime}, A^{\prime}\right),(B, W, A)\right)=\bigoplus_{w: W^{\prime} \longrightarrow W} \mathcal{A}(W)\left(w^{*} A, A^{\prime}\right) \otimes \mathcal{B}(W)\left(B^{\prime}, w^{*} B\right) .
$$

Then we have an isomorphism of categories

$$
\operatorname{Bimod}(\mathcal{A}, \mathcal{B}) \cong \operatorname{Mod}(\mathfrak{r}): M \mapsto\left((B, W, A) \mapsto M^{W}(B, A)\right)
$$

and, by Proposition 3.4, an equivalence of categories

$$
\operatorname{Bimod}(\mathcal{A}, \mathcal{B}) \longrightarrow \operatorname{Bimod}_{\mathcal{U}}^{\mathrm{fib}}(\mathfrak{a}, \mathfrak{b}) .
$$

Then $P_{B, W, A}^{\mathrm{fib}}$ is the image of $\mathfrak{r}(-,(B, W, A))$ under these functors. 
Remark 3.10. With $\mathfrak{t}$ as in Remark 3.8 and $\mathfrak{r}$ as in Remark 3.9, the functor

$$
\Pi^{*}: \operatorname{Bimod}(\mathcal{A}, \mathcal{B}) \longrightarrow \operatorname{Bimod}_{\mathcal{U}}(\mathfrak{a}, \mathfrak{b})
$$

of 3.4 is induced by an underlying linear functor

$$
\Pi: \mathfrak{t} \longrightarrow \mathfrak{r}:(B, u: V \longrightarrow U, A) \longmapsto\left(B, V, u^{*} A\right) .
$$

3.9. Cohomology of natural systems and the bar complex. In [1, the cohomology of $\mathcal{U}$ with values in a natural system $N$ has been defined via a certain complex $\mathbf{C}(\mathcal{U}, N)$. This complex computes in fact

$$
\operatorname{RHom}_{\mathrm{Nat}(\mathcal{U})}(\underline{k}, N),
$$

where $\underline{k}$ is the constant natural system with value $k$ (see [1, Thm. 4.4]). If $N$ is obtained from a presheaf, then this reduces to ordinary presheaf cohomology. I.e.,

Proposition 3.11 ([1, Proposition 8.5]). Let $F \in \operatorname{Pr}(\mathcal{U})$. Then

$$
\mathbf{C}(\mathcal{U}, I F) \cong \mathrm{RHom}_{\operatorname{Pr}(\mathcal{U})}(\underline{k}, F) .
$$

To compute (3.8) one uses the bar resolution $B(\underline{k})$ (see the proof of 1 , Thm. 4.4]) of $\underline{k}$. To define this, let $N=N(\mathcal{U})$ denote the simplicial nerve of $\mathcal{U}$. For $v \in N_{n}$ given by

$$
V_{0} \underset{v_{0}}{\longrightarrow} V_{1} \underset{v_{1}}{\longrightarrow} \cdots \longrightarrow V_{n-1} \underset{v_{n-1}}{\longrightarrow} V_{n},
$$

we put $|v|=v_{n-1} \ldots v_{1} v_{0}$. For $V \in N_{0}$ we put $|V|=1_{V}$. Then we have

$$
B(\underline{k})_{n}=\bigoplus_{v \in N_{n}} P_{|v|},
$$

where $P_{u}$ stands for the bimodule associated to the projective natural system $k \operatorname{Fact}(\mathcal{U})(u,-)$.

As a complex, $B(\underline{k})_{n}$ is the chain complex of a simplicial object in $\operatorname{Nat}(\mathcal{U})$, which we will denote by $\mathcal{B}(\underline{k})$. The degeneracies (which we will not use) are obtained from the identity maps

$$
\sigma_{i}^{v}: P_{|v|} \longrightarrow P_{\left|\sigma_{i}(v)\right|}=P_{|v|}
$$

The boundary maps $\partial_{i}$ are obtained from maps

$$
\partial_{i}^{v}: P_{|v|} \longrightarrow P_{\left|\partial_{i}(v)\right|},
$$

which we now define. For $i$ different from 0 and $n,\left|\partial_{i}(v)\right|=|v|$, and the map $\partial_{i}^{v}$ is the identity. For $i=0$ and $i=n, \partial_{0}^{v}$ and $\partial_{n}^{v}$ are obtained from the following maps: $\partial_{i}(v) \rightarrow v$ in $\operatorname{Fact}(\mathcal{U})$ (using the contravariant dependence of $P_{u}$ on $u$ ), i.e.,

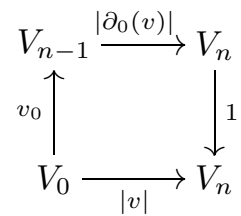

and

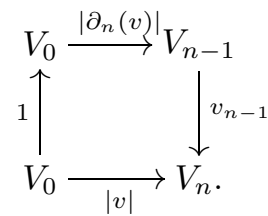

Remark 3.12. If we consider natural systems as a special instance of bimodules as in Remark 3.6, then the complex $\mathbf{C}(\mathcal{U}, N)$ is readily seen to coincide with the Hochschild complex defined in [5]. 
3.10. Resolutions. Now we return to our standard settings: $\mathfrak{a}, \mathfrak{b}$ are fibered $\mathcal{U}$ graded categories equipped with a normalized cleavage, $W \in \mathcal{U}$ and $A \in \mathfrak{a}_{W}$, $B \in \mathfrak{b}_{W}$. The following is our key technical result.

Lemma 3.13. Consider the constant natural system $\underline{k}$ on $\mathcal{U} / W$. We have

$$
L \Phi_{A, B, !}(\underline{k})=\Phi_{A, B, !}(\underline{k})=P_{B, W, A}^{\mathrm{fib}} .
$$

Proof. By definition, $L \Phi_{A, B, !}(\underline{k})=\Phi_{A, B, !}(B(\underline{k}))$. We will define an augmentation map $\left.\epsilon: \Phi_{A, B, !}(\mathcal{B}(\underline{k}))_{0}\right) \longrightarrow P_{B, W, A}^{\mathrm{fib}}$ and for every $u^{\prime}: V^{\prime} \rightarrow U^{\prime}, B^{\prime} \in \mathfrak{b}_{V^{\prime}}, A^{\prime} \in \mathfrak{a}_{U^{\prime}}$ we will show that the augmented simplicial abelian group $\left(\Phi_{A, B, !}(\mathcal{B}(\underline{k}))_{u^{\prime}}\left(B^{\prime}, A^{\prime}\right), \epsilon\right)$ is contractible. As the chain complex associated to $\Phi_{A, B, !}(\mathcal{B}(\underline{k}))$ is $\Phi_{A, B, !}(B(\underline{k}))$ this proves the claim.

We need to give a detailed description of the simplicial object $\Phi_{A, B, !}(\mathcal{B}(\underline{k}))$. Put $N=N(\mathcal{U} / W)$. We write the elements of $N_{n}$ as $(v, f)$, where $v$ represents an element of $N(\mathcal{U})_{n}$,

$$
V_{0} \underset{v_{0}}{\longrightarrow} V_{1} \underset{v_{1}}{\longrightarrow} \cdots \longrightarrow V_{n-1} \underset{v_{n-1}}{\longrightarrow} V_{n},
$$

and $f$ is a map $V_{n} \rightarrow W$. Then

$$
\sigma_{i}(v, f)=\left(\sigma_{i}(v), f\right)
$$

and

$$
\partial_{i}(v, f)= \begin{cases}\left(\partial_{i} v, f\right) & \text { if } i \neq n, \\ \left(\partial_{n} v, v_{n-1} f\right) & \text { if } i=n .\end{cases}
$$

Combining (3.9) with Lemma 3.7(1) we obtain

$$
\Phi_{A, B, !}\left(\mathcal{B}\left(\underline{k}_{n}\right)\right)=\bigoplus_{(v, f) \in N_{n}} P_{(f|v|) * B,|v|, f^{*} A} .
$$

The explicit description of $\mathcal{B}(\underline{k})$ given in $\$ 3.9$ combined with Lemma 3.7(2) allows us to describe the degeneracies and boundary maps in $\Phi_{A, B, !}(\mathcal{B}(\underline{k}))$.

The degeneracies on $\Phi_{A, B, !}(\mathcal{B}(\underline{k}))$ (which we do not use) are obtained from the identity maps

$$
\sigma_{i}^{v}: P_{(f|v|)^{*} B,|v|, f^{*} A} \longrightarrow P_{\left(f\left|\sigma_{i}(v)\right|\right)^{*} B,\left|\sigma_{i}(v)\right|, f^{*} A}=P_{(f|v|)^{*} B,|v|, f^{*} A} .
$$

Likewise the boundary maps $\partial_{i}$ for $i \neq 0, n$ are obtained from the identity maps

$$
\partial_{i}^{v}: P_{(f|v|) * B,|v|, f^{*} A} \longrightarrow P_{\left(f\left|\partial_{i}(v)\right|\right)^{*} B,\left|\partial_{i}(v)\right|, f^{*} A}=P_{(f|v|) * B,|v|, f^{*} A}
$$

$\partial_{0}$ is obtained from the maps

$$
\partial_{0}^{v}: P_{(f|v|) * B,|v|, f * A} \longrightarrow P_{\left(f\left|\partial_{0} v\right|\right) * B,\left|\partial_{0} v\right|, f^{*} A},
$$

where with the notation of (3.6) we have $\partial_{0}^{v}=\delta_{v_{0}}^{r}$.

Similarly $\partial_{n}$ is obtained from the maps

$$
\partial_{n}^{v}: P_{(f|v|)^{*} B,|v|, f^{*} A} \longrightarrow P_{\left(f v_{n-1}\left|\partial_{n} v\right|\right)^{*} B,\left|\partial_{n} v\right|,\left(f v_{n-1}\right)^{*} A},
$$

where with the notation of (3.6) we have $\partial_{n}^{v}=\delta_{v_{n-1}}^{l}$.

Now we discuss the case $n=1$. The boundary maps

$$
\partial_{0}, \partial_{1}: \mathcal{B}(\underline{k})_{1} \longrightarrow \mathcal{B}(\underline{k})_{0}
$$


are obtained from

$$
\begin{gathered}
\partial_{0}^{u}: P_{(f u)^{*} B, u, f^{*} A} \longrightarrow P_{f^{*} B, 1_{U}, f^{*} A}, \\
\partial_{1}^{u}: P_{(f u)^{*} B, u, f^{*} A} \longrightarrow P_{(f u)^{*} B, 1_{V},(f u)^{*} A} .
\end{gathered}
$$

These are respectively given by $\delta_{u}^{r}$ and $\delta_{u}^{l}$.

Let $M$ be a fibered bimodule and fix an element $x \in M_{1_{W}}(B, A)$. Then since $M$ corresponds to a bimodule over the corresponding $k$-linear prestack on $\mathcal{U}$ we have corresponding restricted elements $\rho^{f}(x) \in M_{1_{U}}\left(f^{*} B, f^{*} A\right)$ and $\rho^{f u}(x) \in M_{1_{V}}\left((f u)^{*} B,(f u)^{*} A\right)$. One checks that the following identity holds in $M_{u}\left((f u)^{*} B, f^{*} A\right)$ :

$$
\rho^{f}(x) \cdot \delta_{u, f^{*} B}=\delta_{u, f^{*} A} \cdot \rho^{f u}(x)
$$

Let

$$
\epsilon_{x}^{f}: P_{f^{*} A, 1_{U}, f^{*} B} \longrightarrow M
$$

correspond to $\rho^{f}(x) \in M_{1_{U}}(B, A)$. Then (3.10) combined with the discussion in $\$ 3.7$ yields a commutative diagram

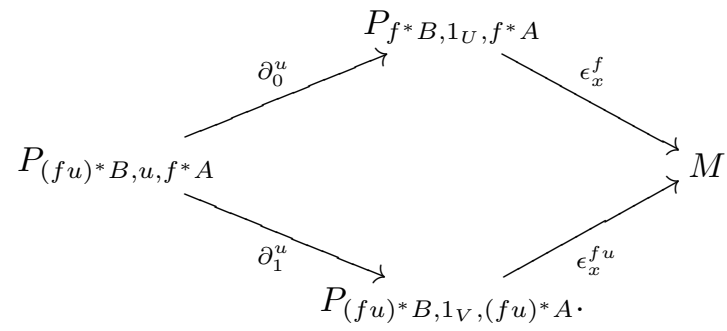

We use this to define an augmentation

$$
\epsilon: \Phi_{A, B, !}\left(\mathcal{B}(\underline{k})_{0}\right) \longrightarrow P_{B, W, A}^{\mathrm{fib}} .
$$

For every $f: U \longrightarrow W$ in $N_{0}(\mathcal{U} / W)$, we have to define a map

$$
\epsilon^{f}: P_{f * B, 1_{U}, f * A} \longrightarrow P_{B, W, A}^{\mathrm{fib}} .
$$

Let $M=P_{A, W, B}^{\mathrm{fib}}$. Then

$$
P_{A, W, B}^{\mathrm{fib}}\left(A, 1_{W}, B\right)=\operatorname{Bimod}_{\mathcal{U}}(\mathfrak{a}, \mathfrak{b})\left(P_{B, W, A}^{\mathrm{fib}}, P_{A, W, B}^{\mathrm{fib}}\right)
$$

contains a canonical element $x$ given by the identity morphism. We put $\epsilon^{f}=\epsilon_{x}^{f}$. Then by 3.11 we obtain

$$
\epsilon^{f} \partial_{0}^{u}=\epsilon^{f u} \partial_{1}^{u}
$$

Now we show that for every $u^{\prime}: V^{\prime} \rightarrow U^{\prime}$ in $\mathcal{U}$ and for every $B^{\prime} \in \mathfrak{b}_{V^{\prime}}, A^{\prime} \in \mathfrak{a}_{U^{\prime}}$ the augmented simplicial object $S \stackrel{\text { def }}{=}\left(\Phi_{A, B, !}(\mathcal{B}(\underline{k}))_{u^{\prime}}\left(B^{\prime}, A^{\prime}\right), \epsilon\right)$ is left contractible in the sense of $[12, \S 8.4 .6]$. That is, we have to produce contracting homotopies

$$
h_{n}: S_{n} \longrightarrow S_{n+1}
$$

for $n \geq-1$ such that

$$
\begin{aligned}
& \partial_{0} h_{n}=\operatorname{Id}_{S_{n}}, \\
& \partial_{i} h_{n}=h_{n-1} \partial_{i-1} \quad(i>0),
\end{aligned}
$$

where $\partial_{0}: S_{0} \rightarrow S_{-1}$ should be interpreted as $\epsilon$. 
We start by defining a map

$$
h_{-1}: S_{-1}=\left(P_{B, W, A}^{\mathrm{fib}}\right)_{u^{\prime}}\left(B^{\prime}, A^{\prime}\right) \longrightarrow \bigoplus_{f: U \rightarrow W \in N_{0}}\left(P_{f^{*} B, 1_{U}, f^{*} A}\right)_{u^{\prime}}\left(B^{\prime}, A^{\prime}\right)=S_{0} .
$$

We have (see 93.8 )

$$
\left(P_{B, W, A}^{\mathrm{fib}}\right)_{u^{\prime}}\left(B^{\prime}, A^{\prime}\right): \bigoplus_{f: V^{\prime} \rightarrow W} \mathfrak{b}_{1_{V^{\prime}}}\left(B^{\prime}, f^{*} B\right) \otimes \mathfrak{a}_{u^{\prime}}\left(f^{*} A, A^{\prime}\right)
$$

and

$$
\left(P_{f^{*} B, 1_{U}, f^{*} A}\right)_{u^{\prime}}\left(B^{\prime}, A^{\prime}\right)=\bigoplus_{p q=u^{\prime}} \mathfrak{b}_{q}\left(B^{\prime}, f^{*} B\right) \otimes \mathfrak{a}_{p}\left(f^{*} A, A^{\prime}\right) .
$$

We send the part of $\left(P_{B, W, A}^{\mathrm{fib}}\right)_{u^{\prime}}\left(B^{\prime}, A^{\prime}\right)$ given by

$$
\mathfrak{b}_{1_{V^{\prime}}}\left(B^{\prime}, f^{*} B\right) \otimes \mathfrak{a}_{u^{\prime}}\left(f^{*} A, A^{\prime}\right)
$$

via the identity morphism to the identical part of $P_{f^{*} B, 1_{V^{\prime}}, f^{*} A}$ corresponding to $q=1_{V^{\prime}}, p=u^{\prime}$.

Before continuing we will check

$$
\epsilon h_{-1}=\operatorname{Id}_{S_{-1}} .
$$

To do this we need to make explicit $\epsilon: S_{0} \rightarrow S_{-1}$. One verifies that $\epsilon$ sends an element

$$
b \otimes a \in \mathfrak{b}_{q}\left(B^{\prime}, f^{*} B\right) \otimes \mathfrak{a}_{p}\left(f^{*} A, A^{\prime}\right) \subset\left(P_{f^{*} B, 1_{U}, f^{*} A}\right)_{u^{\prime}}\left(B^{\prime}, A^{\prime}\right)
$$

to the element of

$$
\mathfrak{b}_{1_{V^{\prime}}}\left(B^{\prime},(f q)^{*} B\right) \otimes \mathfrak{a}_{u^{\prime}}\left((f q)^{*} A, A^{\prime}\right) \subset\left(P_{B, W, A}^{\mathrm{fib}}\right)_{u^{\prime}}\left(B^{\prime}, A^{\prime}\right)
$$

given by

$$
\delta_{q, f^{*} B}^{-1} \cdot b \otimes a \cdot \delta_{q, f^{*} A}
$$

where we have committed a slight abuse of notation (cartesian arrows are not invertible but left multiplying with them is). So if $q=1_{V^{\prime}}$, then $\epsilon(b \otimes a)=b \otimes a$. From this one deduces immediately that $\epsilon h_{-1}=\operatorname{Id}_{S_{-1}}$.

For use below we also compute the other composition $h_{-1} \epsilon$. Let $b \otimes a$ be as above. Then

$$
\begin{aligned}
h_{-1} \epsilon(b \otimes a) & =h_{-1}\left(\delta_{q, f^{*} B}^{-1} \cdot b \otimes a \cdot \delta_{q, f^{*} A}\right) \\
& =\delta_{q, f^{*} B}^{-1} \cdot b \otimes a \cdot \delta_{q, f^{*} A} .
\end{aligned}
$$

Next we define $h_{n}$. This is a map

$$
h_{n}: \bigoplus_{(u, f) \in N_{n}}\left(P_{(f|u|)^{*} B,|u|, f^{*} A}\right)_{u^{\prime}}\left(B^{\prime}, A^{\prime}\right) \longrightarrow \bigoplus_{(u, f) \in N_{n+1}}\left(P_{(f|u|)^{*} B,|u|, f^{*} A}\right)_{u^{\prime}}\left(B^{\prime}, A^{\prime}\right) .
$$

The summand on the left attached to

$$
(u, f)=\left(U_{0} \longrightarrow \ldots \longrightarrow U_{n} \longrightarrow W\right) \in N_{n}(\mathcal{U} / W)
$$

is given by

$$
\bigoplus_{u^{\prime}=p|u| q} \mathfrak{b}_{q}\left(B^{\prime},(f|u|)^{*} B\right) \otimes \mathfrak{a}_{p}\left(f^{*} A, A^{\prime}\right)
$$

We send an element $b \otimes a \in \mathfrak{b}_{q}\left(B^{\prime},(f|u|)^{*} B\right) \otimes \mathfrak{a}_{p}\left(f^{*} A, A^{\prime}\right)$ to the element of

$$
\mathfrak{b}_{1_{V^{\prime}}}\left(B^{\prime},(f|u q|)^{*} B\right) \otimes \mathfrak{a}_{p}\left(f^{*} A, A^{\prime}\right) \subset\left(P_{(f|u q|)^{*} B,|u q|, f^{*} A}\right)_{u^{\prime}}\left(B^{\prime}, A^{\prime}\right)
$$


given by $\delta_{q,(f|u|) * B}^{-1} b \otimes a$. Here $\left(P_{(f|u q|)^{*} B,|u q|, f^{*} A}\right)_{u^{\prime}}$ is considered as being attached to

$$
\left(\bullet \stackrel{q}{\rightarrow} U_{0} \longrightarrow \ldots \longrightarrow U_{n} \longrightarrow W\right) \in N_{n+1}(\mathcal{U} / W) .
$$

We now check that $h$ has the required properties. Let $b \otimes a$ be as in the previous paragraph. We compute for $n \geq 0$,

$$
\begin{aligned}
\partial_{0} h_{n}(b \otimes a) & =\delta_{q}^{r}\left(\delta_{q,(f|u|)^{*} B}^{-1} b \otimes a\right) \\
& =\delta_{q,(f|u|)^{*} B} \delta_{q,(f|u|)^{*} B}^{-1} b \otimes a \\
& =b \otimes a .
\end{aligned}
$$

If $0<i<n+1$, then

$$
\partial_{i} h_{n}(b \otimes a)=\delta_{q,(f|u|) * B}^{-1} b \otimes a
$$

and if $i=n+1, n>0$, then

$$
\begin{aligned}
\partial_{n+1} h_{n}(b \otimes a) & =\delta_{u_{n-1}}^{l}\left(\delta_{q,(f|u|) * B}^{-1} b \otimes a\right) \\
& =\delta_{q,(f|u|)^{*} B}^{-1} b \otimes a \delta_{u_{n-1}, f^{*} A} .
\end{aligned}
$$

If $n=0, i=1$, then the same computation yields

$$
\partial_{1} h_{0}(b \otimes a)=\delta_{q, f^{*} B}^{-1} b \otimes a \delta_{q, f^{*} A} .
$$

Now assume $n>0$. We compute

$$
\begin{aligned}
h_{n-1} \partial_{0}(b \otimes a) & =h_{n} \delta_{u_{0}}^{r}(b \otimes a) \\
& =h_{n}\left(\delta_{u_{0},\left(f\left|\partial_{0} u\right|\right)^{*} B} b \otimes a\right) \\
& =\delta_{u_{0} q,\left(f\left|\partial_{0} u\right|\right)^{*} B}^{-1} \delta_{u_{0},\left(f\left|\partial_{0} u\right|\right)^{*} B} b \otimes a \\
& =\delta_{q,(f|u|)^{*} B}^{-1} b \otimes a
\end{aligned}
$$

and for $0<i<n$,

$$
\begin{aligned}
h_{n-1} \partial_{i}(b \otimes a) & =h_{n-1}(b \otimes a) \\
& =\delta_{q,(f|u|)^{*} B}^{-1} b \otimes a .
\end{aligned}
$$

Finally

$$
\begin{aligned}
h_{n-1} \partial_{n}(b \otimes a) & =h_{n} \delta_{u_{n-1}}^{l}(b \otimes a) \\
& =h_{n}\left(b \otimes a \delta_{u_{n-1}, f^{*} A}\right) \\
& =\delta_{q,(f|u|)^{*} B}^{-1} b \otimes a \delta_{u_{n-1}, f^{*} A} .
\end{aligned}
$$

The conditions (3.12) now follow by combining (3.13), (3.14), (3.15), (3.16), (3.17), (3.18), (3.19), (3.20) and (3.21).

\section{THE COHOMOLOGY COMPARISON THEOREM}

4.1. Main result. Let $\mathcal{A}$ and $\mathcal{B}$ be $k$-linear prestacks on $\mathcal{U}$ with associated fibered graded categories $\mathfrak{a}$ and $\mathfrak{b}$. The following is our main result.

Theorem 4.1. The functor $\Pi^{*}: \operatorname{Bimod}(\mathcal{A}, \mathcal{B}) \longrightarrow \operatorname{Bimod}_{\mathcal{U}}(\mathfrak{a}, \mathfrak{b})$ induces a fully faithful functor

$$
\Pi^{*}: D(\operatorname{Bimod}(\mathcal{A}, \mathcal{B})) \longrightarrow D\left(\operatorname{Bimod}_{\mathcal{U}}(\mathfrak{a}, \mathfrak{b})\right)
$$


In particular, for $M, N \in \operatorname{Bimod}(\mathcal{A}, \mathcal{B})$, there are isomorphisms

$$
\operatorname{Ext}_{\operatorname{Bimod}(\mathcal{A}, \mathcal{B})}^{i}(M, N) \cong \operatorname{Ext}_{\operatorname{Bimod} \mathcal{U}(\mathfrak{a}, \mathfrak{b})}^{i}\left(\Pi^{*} M, \Pi^{*} N\right)
$$

for all $i$.

Proof. According to Proposition 3.4 we may $\operatorname{replace} \operatorname{Bimod}(\mathcal{A}, \mathcal{B})$ by $\operatorname{Bimod}_{\mathcal{U}}^{\mathrm{fib}}(\mathfrak{a}, \mathfrak{b})$. As before we denote the inclusion $\operatorname{Bimod}_{\mathcal{U}}^{\mathrm{fib}}(\mathfrak{a}, \mathfrak{b}) \rightarrow \operatorname{Bimod}_{\mathcal{U}}(\mathfrak{a}, \mathfrak{b})$ by $I$.

For all objects $M, N \in D\left(\operatorname{Bimod}_{\mathcal{U}}^{\text {fib }}(\mathfrak{a}, \mathfrak{b})\right)$ we have to prove that the canonical map

$$
\operatorname{RHom}_{\operatorname{Bimod}_{\mathcal{U}}^{\mathrm{fib}}(\mathfrak{a}, \mathfrak{b})}(M, N) \longrightarrow \operatorname{RHom}_{\operatorname{Bimod}_{\mathcal{U}}(\mathfrak{a}, \mathfrak{b})}(I M, I N)
$$

is an isomorphism. We claim that it is sufficient to check this for $M=P_{B, W, A}^{\mathrm{fib}}$.

To see this note that the projective objects $P_{A, W, B}^{\mathrm{fib}}$ form a system of compact generators for $D\left(\operatorname{Bimod}_{\mathcal{U}}^{\mathrm{fib}}(\mathfrak{a}, \mathfrak{b})\right)$. If we fix $N$, then the $M$ for which (4.1) is an isomorphism is a triangulated subcategory of $D\left(\operatorname{Bimod}^{\mathrm{fib}}(\mathfrak{a}, \mathfrak{b})\right)$. If this subcategory contains the generators $P_{B, W, A}^{\mathrm{fib}}$, then it is everything.

We now assume $M=P_{B, W, A}^{\mathrm{fib}}$. Then we have

$$
\operatorname{RHom}_{\operatorname{Bimod}_{\mathcal{U}}^{\mathrm{fib}}(\mathfrak{a}, \mathfrak{b})}\left(P_{B, W, A}^{\mathrm{fib}}, N\right)=N_{1_{W}}(B, A) .
$$

On the other hand we have

$$
\begin{aligned}
& \mathrm{RHom}_{\operatorname{Bimod}_{\mathcal{U}}(\mathfrak{a}, \mathfrak{b})}\left(I P_{A, W, B}^{\mathrm{fib}}, I N\right) \\
& =\operatorname{RHom}_{\operatorname{Bimod}_{\mathcal{U}}(\mathfrak{a}, \mathfrak{b})}\left(L \Phi_{A, B, \underline{k}, \underline{k}, I N) \quad \text { (Lemma 3.13) }}\right. \\
& =\operatorname{RHom}_{\mathrm{Nat}(\mathcal{U} / W)}\left(\underline{k}, \Phi_{A, B}^{*} I N\right) \quad \text { (Adjointness) } \\
& =\operatorname{RHom}_{\mathrm{Nat}(\mathcal{U} / W)}\left(\underline{k}, I \Psi_{A, B}^{*} N\right) \quad \text { (Prop. 3.5) } \\
& =\mathrm{RHom}_{\operatorname{Pr}(\mathcal{U} / W)}\left(\underline{k}, \Psi_{A, B}^{*} N\right) \quad \text { (Prop. 3.11) } \\
& =\left(\Psi_{A, B}^{*} N\right)\left(1_{W}\right) \quad\left(1_{W} \text { is the final object of } \mathcal{U} / W\right) \\
& =N_{1_{W}}(B, A)
\end{aligned}
$$

which is the same.

The following corollary was announced in [5]:

Corollary 4.2. Let $\mathcal{A}$ be a $k$-flat $k$-linear prestack on $\mathcal{U}$ with associated fibered category $\mathfrak{a}$, and let $\mathbf{C}(\mathcal{A})=\mathbf{C}(\mathfrak{a})$ be the Hochschild complex of $\mathcal{A}$ defined in [5]. Then there is a quasi-isomorphism

$$
\mathrm{C}(\mathcal{A}) \cong \operatorname{RHom}_{\operatorname{Bimod}(\mathcal{A})}(\mathcal{A}, \mathcal{A}) .
$$

Proof. It was shown in [5, Proposition 3.13] that

$$
\mathbf{C}(\mathfrak{a}) \cong \operatorname{RHom}_{\operatorname{Bimod} \mathcal{U}(\mathfrak{a})}(\mathfrak{a}, \mathfrak{a}) .
$$

To obtain the desired result, it suffices to apply Theorem 4.1 with $\mathfrak{b}=\mathfrak{a}$ and note that $\Pi^{*}(\mathcal{A})=\mathfrak{a}$. 


\section{THE SPECIAL COHOMOLOGY COMPARISON THEOREM REVISITED}

In this section, we deduce the original Special Cohomology Comparison Theorem 4 from Theorem 4.1

If $\mathfrak{a}$ is a $k$-linear category, then we define $\overline{\mathfrak{a}}$ to be the endomorphism ring of the generator $P=\bigoplus_{A} \mathfrak{a}(-, A)$ of $\operatorname{Mod}(\mathfrak{a})$. I.e. by Yoneda,

$$
\overline{\mathfrak{a}}=\prod_{B \in \mathfrak{a}} \bigoplus_{A \in \mathfrak{a}} \mathfrak{a}(B, A)
$$

Elements of $\overline{\mathfrak{a}}$ can be represented by column-finite matrices with (row, column) indices given by $(A, B)$. We denote the idempotent corresponding to $1_{A} \in \mathfrak{a}(A, A)$ by $e_{A}$.

Lemma 5.1. Let $\mathfrak{a}$ and $\mathfrak{b}$ be linear categories. Then the functor

$$
D(\operatorname{Bimod}(\mathfrak{a}, \mathfrak{b})) \longrightarrow D(\operatorname{Bimod}(\overline{\mathfrak{a}}, \overline{\mathfrak{b}})): M \mapsto \prod_{B \in \mathfrak{a}} \bigoplus_{A \in \mathfrak{a}} M(B, A)
$$

is fully faithful.

Proof. This follows from the fact that a left inverse is given by

$$
N \mapsto\left((B, A) \mapsto e_{A} N e_{B}\right) .
$$

Let $\mathcal{U}$ be a poset. If $\mathfrak{a}$ is a $\mathcal{U}$-graded category, then we denote by $\tilde{\mathfrak{a}}$ the underlying $k$-linear category of $\mathfrak{a}$. More precisely

for $B_{V} \in \mathfrak{b}_{V}, A_{U} \in \mathfrak{a}_{U}$.

$$
\tilde{\mathfrak{a}}\left(B_{V}, A_{U}\right)= \begin{cases}\mathfrak{a}_{V \leq U}\left(B_{V}, A_{U}\right) & \text { if } V \leq U, \\ 0 & \text { otherwise, }\end{cases}
$$

Lemma 5.2. The functor

$$
D\left(\operatorname{Bimod}_{\mathcal{U}}(\mathfrak{a}, \mathfrak{b})\right) \longrightarrow D(\operatorname{Bimod}(\tilde{\mathfrak{a}}, \tilde{\mathfrak{b}})): N \longrightarrow \tilde{N}
$$

with

$$
\tilde{N}\left(B_{V}, A_{U}\right)= \begin{cases}N_{V \leq U}\left(B_{V}, A_{U}\right) & \text { if } V \leq U, \\ 0 & \text { otherwise, }\end{cases}
$$

for $B_{V} \in \mathfrak{b}_{V}, A_{U} \in \mathfrak{a}_{U}$ is fully faithful.

Proof. A left inverse is given by associating to $N \in D(\operatorname{Bimod}(\tilde{\mathfrak{a}}, \tilde{\mathfrak{b}}))$ the object $\check{N}$ in $D\left(\operatorname{Bimod}_{\mathcal{U}}(\mathfrak{a}, \mathfrak{b})\right)$ such that $\check{N}_{V \leq U}\left(B_{V}, A_{U}\right)=N\left(B_{V}, A_{U}\right)$. In other words the left inverse simply forgets the values of $N\left(B_{V}, A_{U}\right)$ for $V \not \leq U$.

If $\mathcal{A}$ is a $k$-linear prestack on $\mathcal{U}$, then we write $\mathcal{A} !=\overline{\tilde{a}}$, where $\mathfrak{a}$ is the $\mathcal{U}$-graded category associated to $\mathcal{A}$. Concretely

$$
\mathcal{A} !=\prod_{V \in \mathcal{U}, A_{V} \in \mathcal{A}(V)} \bigoplus_{V \leq U, A_{U} \in \mathcal{A}(U)} \mathcal{A}(V)\left(A_{V},\left.A_{U}\right|_{V}\right) .
$$

Theorem 5.3. Let $\mathcal{A}$ and $\mathcal{B}$ be $k$-linear prestacks on a poset $\mathcal{U}$. The canonical functor

with

$$
(-) !: \operatorname{Bimod}(\mathcal{A}, \mathcal{B}) \longrightarrow \operatorname{Bimod}(\mathcal{A} !, \mathcal{B} !): M \longrightarrow M !
$$

$$
M !=\prod_{V \in \mathcal{U}, B_{V} \in \mathcal{B}(V)} \bigoplus_{V \leq U, A \in \mathcal{A}(U)} M(V)\left(B_{V},\left.A_{U}\right|_{V}\right)
$$

induces a fully faithful functor between the respective derived categories. 
Proof. Let $\mathfrak{a}, \mathfrak{b}$ be the $\mathcal{U}$-graded categories associated to $\mathcal{A}, \mathcal{B}$.

The functor $D(-)$ ! is the composition of the following fully faithful embeddings:

$$
\begin{aligned}
D(\operatorname{Bimod}(\mathcal{A}, \mathcal{B})) \stackrel{\text { Thm. } \stackrel{4.1}{\longrightarrow}}{\longrightarrow} D\left(\operatorname{Bimod}_{\mathcal{U}}(\mathfrak{a}, \mathfrak{b})\right) \\
\stackrel{\text { Lem. } \underline{5.2}}{\longrightarrow} D(\operatorname{Bimod}(\tilde{\mathfrak{a}}, \tilde{\mathfrak{b}})) \stackrel{\text { Lem. } 5.1}{\longrightarrow} D(\operatorname{Bimod}(\overline{\tilde{\mathfrak{a}}}, \overline{\tilde{\mathfrak{b}}})) .
\end{aligned}
$$

The following corollary is the Special Cohomology Comparison Theorem in the sense of Gerstenhaber and Schack.

Corollary 5.4. Let $\mathcal{A}$ be a presheaf of k-algebras on a poset $\mathcal{U}$. Put

$$
\mathcal{A} !=\prod_{V \in \mathcal{U}} \bigoplus_{V \leq U} \mathcal{A}(V)
$$

The canonical functor

$$
(-) !: \operatorname{Bimod}(\mathcal{A}) \longrightarrow \operatorname{Bimod}(\mathcal{A} !): M \longrightarrow M !
$$

with

$$
M !=\prod_{V \in \mathcal{U}} \bigoplus_{V \leq U} M(V)
$$

induces a fully faithful functor between the respective derived categories.

Proof. If we view a presheaf as a prestack, then the categories $\mathcal{A}(V)$ contain only one object which is left out of the notation. This gives the simplified form of $\mathcal{A}$ ! and $M$ ! is the statement of this corollary.

\section{Appendix A. Relation with universal localization}

Let $\mathfrak{a}, \mathfrak{b}$ be $\mathcal{U}$-graded fibered categories equipped with a normalized cleavage. Let $u: V \rightarrow U, p: U \rightarrow U^{\prime}, B \in \mathfrak{a}_{V}, A^{\prime} \in \mathfrak{a}_{U^{\prime}}$. The natural transformation between the functors

$$
\begin{aligned}
M & \mapsto M_{u}\left(B, p^{*} A^{\prime}\right), \\
M & \mapsto M_{p u}\left(B, A^{\prime}\right),
\end{aligned}
$$

corresponding to $\delta_{p}^{l}$ is given by left multiplication by $\delta_{p, A^{\prime}}$ (see 3.7). Hence $M$ is fibered if and only if for all $u, p, B, A^{\prime}$ as above $\operatorname{Bimod}_{\mathcal{U}}(\mathfrak{a}, \mathfrak{b})(-, M)$ inverts the corresponding $\delta_{p}^{l}$. Thus if we denote the collections of such morphisms by $\Sigma$, then we get

$$
\operatorname{Bimod}_{\mathcal{U}}^{\mathrm{fib}}(\mathfrak{b}, \mathfrak{a})=\left\{M \in \operatorname{Bimod}_{\mathcal{U}}(\mathfrak{b}, \mathfrak{a}) \mid M \text { inverts all morphisms in } \Sigma\right\} .
$$

Using the description of $\operatorname{Bimod}_{\mathcal{U}}(\mathfrak{b}, \mathfrak{a})$ as a module category in Remark 3.8 we also obtain

$$
\operatorname{Bimod}_{\mathcal{U}}^{\mathrm{fib}}(\mathfrak{b}, \mathfrak{a})=\operatorname{Mod}\left(\Sigma^{-1} \mathfrak{t}\right)
$$

This shows that the relation between $\operatorname{Bimod}_{\mathcal{U}}^{\mathrm{fib}}(\mathfrak{b}, \mathfrak{a})$ and $\operatorname{Bimod}_{\mathcal{U}}(\mathfrak{b}, \mathfrak{a})$ is controlled by a universal localization (which is given by $\Pi: \mathfrak{t} \longrightarrow \mathfrak{r}$ as in Remark 3.10). Unfortunately this is not an Ore localization. The homological behavior of a general universal localization seems difficult to understand. See e.g. 9, 10] for partial results.

Theorem 4.1 may be interpreted as saying that $\Sigma^{-1} \mathfrak{t} / \mathfrak{t}$ is stably flat (see [8]). This implies for example that the $K$-theory of the categories of bimodules and fibered bimodules are related by a long exact sequence [7, Thm. 0.1]. 


\section{REFERENCES}

[1] H. J. Baues and G. Wirsching, Cohomology of small categories, J. Pure Appl. Algebra 38 (1985), no. 2-3, 187-211. MR814176 (87g:18013)

[2] M. Gerstenhaber and S. D. Schack, On the deformation of algebra morphisms and diagrams, Trans. Amer. Math. Soc. 279 (1983), no. 1, 1-50. MR704600(85d:16021)

[3] _ Algebraic cohomology and deformation theory, Deformation theory of algebras and structures and applications (Il Ciocco, 1986), NATO Adv. Sci. Inst. Ser. C Math. Phys. Sci., vol. 247, Kluwer Acad. Publ., Dordrecht, 1988, pp. 11-264. MR981619 (90c:16016)

[4] _ The cohomology of presheaves of algebras. I. Presheaves over a partially ordered set, Trans. Amer. Math. Soc. 310 (1988), no. 1, 135-165. MR965749 (89k:16052)

[5] W. Lowen, Hochschild cohomology of presheaves as map-graded categories, Internat. Math. Res. Notices 118 (2008), 32pp. MR2449052 (2009i:18015)

[6] W. Lowen and M. Van den Bergh, A local to global spectral sequence for Hochschild cohomology, in preparation.

[7] A. Neeman, Noncommutative localisation in algebraic K-theory. II, Adv. Math. 213 (2007), no. 2, 785-819. MR.2332610 (2008d:19001)

[8] A. Neeman and A. Ranicki, Noncommutative localisation in algebraic K-theory. I, Geom. Topol. 8 (2004), 1385-1425 (electronic). MR2119300 (2005k:19006)

[9] A. Neeman, A. Ranicki, and A. Schofield, Representations of algebras as universal localizations, Math. Proc. Cambridge Philos. Soc. 136 (2004), no. 1, 105-117. MR2034017 (2005b:16031)

[10] A. H. Schofield, Representation of rings over skew fields, London Mathematical Society Lecture Note Series, vol. 92, Cambridge University Press, Cambridge, 1985. MR800853 (87c:16001)

[11] A. Vistoli, Grothendieck topologies, fibered categories and descent theory, Fundamental algebraic geometry, Math. Surveys Monogr., vol. 123, Amer. Math. Soc., Providence, RI, 2005, pp. 1-104. MR2223406

[12] C. Weibel, Cyclic homology for schemes, Proc. Amer. Math. Soc. 124 (1996), no. 6, 16551662. MR1277141(96h:19003)

Departement Wiskunde-Informatica, University of Antwerpen, Middelheimcampus, Middelheimlaan 1, 2020 Antwerp, Belgium

E-mail address: wendy.lowen@ua.ac.be

Department WNi, Hasselt University, Agoralaan, 3590 Diepenbeek, Belgium

E-mail address: michel.vandenbergh@uhasselt.be 